\title{
Incidence, impact and indicators of difficult intubations in the neonatal intensive care unit: a report from the National Emergency Airway Registry for Neonates
}

\begin{abstract}
Taylor Sawyer, ${ }^{1}$ Elizabeth E Foglia, ${ }^{\odot 2}$ Anne Ades, ${ }^{3}$ Ahmed Moussa, ${ }^{4}$ Natalie Napolitano, ${ }^{5}$ Kristen Glass, ${ }^{6}$ Lindsay Johnston, ${ }^{7}$ Philipp Jung, ${ }^{8}$ Neetu Singh, ${ }^{9}$ Bin Huey Quek, ${ }^{10}$ James Barry, ${ }^{11}$ Jeanne Zenge, ${ }^{11}$ Stephen D DeMeo, ${ }^{12}$ Brianna Brei, $^{13}$ Jeanne Krick, ${ }^{13}$ Jae H Kim, ${ }^{14}$ Vinay Nadkarni, ${ }^{15,16}$ Akira Nishisaki, ${ }^{15,16}$ for the National Emergency Airway Registry for Neonates (NEAR4NEOS) investigators
\end{abstract}

For numbered affiliations see end of article.

\section{Correspondence to}

Dr Taylor Sawyer, Department of Pediatrics, School of Medicine, University of Washington, Seattle WA 98195-6320, USA; tlsawyer@uw.edu

Received 4 October 2018 Revised 7 January 2019 Accepted 6 February 2019 Published Online First 22 February 2019

\section{Linked}

- http://dx.doi.org/10.1136/ archdischild-2018-316711

\section{Check for updates}

(C) Author(s) (or their employer(s)) 2019. No commercial re-use. See rights and permissions. Published by BMJ.

To cite: Sawyer T, Foglia EE, Ades A, et al. Arch Dis Child Fetal Neonatal Ed

2019:104:F461-F466.

\section{ABSTRACT}

Objective To determine the incidence, indicators and clinical impact of difficult tracheal intubations in the neonatal intensive care unit (NICU).

Design Retrospective review of prospectively collected data on intubations performed in the NICU from the National Emergency Airway Registry for Neonates.

Setting Ten academic NICUs.

Patients Neonates intubated in the NICU at each of the sites between October 2014 and March 2017

Main outcome measures Difficult intubation was defined as one requiring three or more attempts by a non-resident provider. Patient (age, weight and bedside predictors of difficult intubation), practice (intubation method and medications used), provider (training level and profession) and outcome data (intubation attempts, adverse events and oxygen desaturations) were collected for each intubation.

Results Out of 2009 tracheal intubations, 276 (14\%) met the definition of difficult intubation. Difficult intubations were more common in neonates $<32$ weeks, $<1500 \mathrm{~g}$. The difficult intubation group had a 4.9 odds ratio (OR) for experiencing an adverse event and a 4.2 $\mathrm{OR}$ for severe oxygen desaturation. Bedside screening tests of difficult intubation lacked sensitivity (receiver operator curve $0.47-0.53$ ).

Conclusions Difficult intubations are common in the NICU and are associated with adverse event and severe oxygen desaturation. Difficult intubations occur more commonly in small preterm infants. The occurrence of a difficult intubation in other neonates is hard to predict due to the lack of sensitivity of bedside screening tests.

\section{INTRODUCTION}

Tracheal intubation (TI) is one of the most commonly performed procedures in the neonatal intensive care unit (NICU). ${ }^{1}$ Adverse events, oxygen desaturations and failed attempts are common during the procedure. Tracheal intubation adverse events (TIAEs) occur in $20 \%-40 \%$ of neonatal TIs. $^{2}{ }^{3}$ Severe oxygen desaturations ( $\geq 20 \%$ decrease in blood oxygen saturation $\left(\mathrm{SpO}_{2}\right)$ ) occur in approximately $50 \%$ of neonatal TIs. ${ }^{2}$ Overall, first attempt success

\section{What is already known on this topic?}

- Neonatal intubation is a common and difficult procedure.

- Failed intubations are associated with adverse events.

- The incidence, impact and indicators of difficult intubation in the neonatal intensive care unit (NICU) are not known.

\section{What this study adds?}

- Difficult intubations occur more commonly in the NICU as compared with the paediatric and adult intensive care unit.

- Difficult intubations are associated with a higher occurrence of adverse events and oxygen desaturations.

- Bedside screening tests of difficult intubation have little value to predict the occurrence of a difficult intubation.

rates for neonatal TI are approximately 50\%, with variation depending on the provider type. ${ }^{4-9}$ First attempt success rates are higher when using videolaryngoscopy-ranging from $63 \%$ to $72 \%$ - but are still suboptimal. ${ }^{10}{ }^{11}$ Failed TI has been linked to intraventricular haemorrhage and neurodevelopmental impairment in premature infants. ${ }^{12} 13$ These data suggest that additional work is needed to make neonatal TI safer. ${ }^{14}$ Accurately predicting which neonates will be difficult to intubate may improve safety. Recent studies have examined the utility of fetal biometric parameters to predict difficult TI in periviable neonates at birth. ${ }^{15}$ However, no prior studies have examined the utility of bedside screening tests of difficult intubation in the NICU.

In the adult and paediatric intensive care unit (ICU), a 'difficult intubation' is defined as one requiring three or more laryngoscopic attempts by an experienced provider (eg, not residents in training). ${ }^{1617}$ The reported incidence of difficult TIs 
in the adult ICU is $8 \%-11 \% .{ }^{16}$ The reported incidence of difficult TIs in the paediatric ICU is $9 \% .{ }^{17}$ In the adult and paediatric ICU, patients with difficult TIs have significantly more adverse events and oxygen desaturations than those with non-difficult TIs. The incidence, impact and indicators of difficult TI in the NICU are unknown.

The primary aims of this study were to: (1) determine the incidence of difficult TIs in the NICU, (2) quantify the clinical impact of difficult TIs and (3) examine the utility of bedside screening tests of difficult neonatal TI. We hypothesised that difficult TI would be common in the NICU, that difficult TIs would be associated with TIAEs and oxygen desaturations and that bedside screening tests could predict the occurrence of difficult TI.

\section{METHODS}

\section{Study design}

This was a retrospective cohort study using prospectively collected data from the National Emergency Airway Registry for Neonates (NEAR4NEOS) database. NEAR4NEOS was established in 2014 with the goal to improve neonatal intubation safety and quality. There were 10 academic NICUs contributing data to the NEAR4NEOS at the time of the study. The current study includes data on all neonates who underwent TI in the NICUs at each of the sites between 1 October 2014 and 31 March 2017.

\section{Data collection}

A standardised NEAR4NEOS data collection tool was used to collect patient, practice, provider and outcome data on all neonatal TIs performed at each participating NICU. The institutional review board at each participating site approved the study or granted a waiver of informed parental consent for data collection and analysis. Standardised NEAR4NEOS operational definitions were used to ensure consistency of data capture across the participating sites. Each site developed a site-specific compliance plan to ensure TI capture of greater than 95\%, as well as accurate and timely data entry. Patient data included gestational age and weight (both at birth and on the day of TI), comorbidities, the indication for TI and the presence or absence of bedside screening tests for difficult TI (reviewed below). Practice data included the method of intubation and all medications used. Provider data included the laryngoscopist's training level and profession. Outcome data included the number of intubation attempts, success or failure for each attempt, TIAEs (defined below) and changes in oxygen saturations $(\mathrm{SpO} 2)$ during TI. These data were collected by the care team at the time of TI on a NEAR4NEOS data form. These data were then entered into a password-protected secure Research Electronic Data Capture system hosted by the Data Coordinating Centre at the Children's Hospital of Philadelphia. ${ }^{18}$

\section{Inclusion and exclusion criteria}

Only TIs in the NICU were included in the analyses. Intubations performed in the delivery room were excluded due to difficulty in evaluating bedside screening tests of difficult TI at the time of birth, as well as limitations in collecting the oxygen saturation data in that group. TIs for elective endotracheal tube changes in the NICU were also excluded. For TIs requiring more than one method of approach (defined as 'Course' in NEAR4NEOS operational definitions), only the first course was included in the analyses.

\section{Difficult TI definition}

We defined a 'difficult neonatal TI' as one requiring three or more intubation attempts by a non-resident provider. This means that a 'difficult TI' would require a minimum of two failed attempts by non-resident providers (with the third attempt being successful). This definition was based on the standard definitions of difficult TI used in the adult and paediatric ICU. ${ }^{16}{ }^{17}$ There was no common algorithm for the escalation of TI approach (eg, change from direct laryngoscopy to videolaryngoscopy) among participating centres. When the first course (method of approach) failed, or non-laryngoscopy approach was used (such as fibreoptic bronchoscopy), the TI was also considered as difficult neonatal TI.

\section{TI adverse events}

TIAEs were classified into two categories: severe and non-severe. $^{2}$ Severe TIAEs included oesophageal intubation with delayed recognition, emesis with witnessed aspiration, hypotension requiring intervention (fluid and/or vasopressors), laryngospasm, malignant hyperthermia, pneumothorax/pneumomediastinum and cardiac arrest. Cardiac arrest was defined as loss of perfusion, or severe bradycardia, requiring chest compressions for $\geq 1 \mathrm{~min}$.

Non-severe TIAEs included oesophageal intubation with immediate recognition, mainstem bronchial intubation, epistaxis, lip trauma, gum or oral trauma, emesis without aspiration, hypertension requiring therapy, pain and/or agitation requiring additional medication and causing a delay in intubation and dysrhythmia. Dysrhythmia included bradycardia to less than 60 beats per minute or arrhythmia requiring treatment.

Oxygen desaturations during TI were determined using the highest $\mathrm{SpO}_{2}$ measured immediately prior to intubation (eg, after preoxygenation) and the lowest $\mathrm{SpO}_{2}$ during the intubation. The NEAR4NEOS programme defines 'severe' oxygen desaturation as $\mathrm{a} \geq 20 \%$ decrease in $\mathrm{SpO}_{2}$ from the pre-TI value. ${ }^{2}$ If either the highest or lowest $\mathrm{SpO}_{2}$ were not recorded, those data were excluded from the analysis.

\section{Bedside screening tests of difficult intubation}

A predefined set of bedside screening tests of difficult TI were included on the NEAR4NEOS data collection form. The predictors were based on those used in the adult and paediatric ICU populations. ${ }^{1617}$ The presence, or absence, of each screening test in the neonate undergoing TI was determined at the time of TI by the intubating team and was recorded on the NEAR4NEOS data form. The screening tests included: a known prior history of difficult airway, upper airway obstruction, limited mouth opening, limited neck extension, micrognathia, midfacial hypoplasia, cleft palate and a short thyromental distance. Where possible, objective measures of the bedside screening tests were used, including, for example, upper airway obstruction defined as an anatomical barrier to visualise glottic opening, widest mouth opening defined as how many patient's fingers fit between gum/incisors and thyromental space defined as how many patient's fingers fit between chin and thyroid cartilage.

\section{Statistical analysis}

Descriptive statistics were presented as numbers and percentages. Non-parametric data were presented as a median and IQRs. The relationship between patient demographics and the occurrence of a difficult TI was analysed using a Wilcoxon rank-sum test. The relationship between 


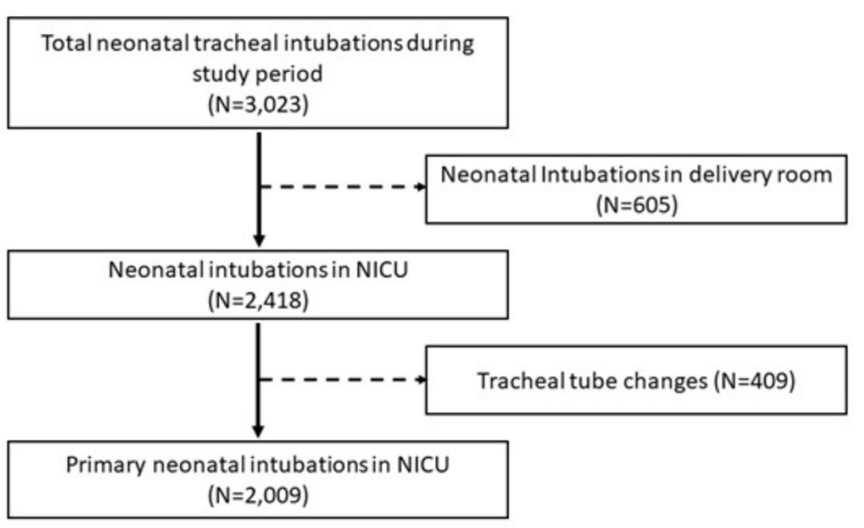

Figure 1 Study flow diagram. NICU, neonatal intensive care unit.

the patient, provider and practice characteristics with the occurrence of difficult TI was analysed using univariate analysis with the $\chi^{2}$, or Fisher's exact test for dichotomous variables, or Wilcoxon rank-sum test for numeric variables. The utility of bedside screening tests of difficult TI was evaluated by calculating the sensitivity, specificity, positive predictive value, negative predictive value and area under the receiver operator curve (ROC) for each. The clinical impact of difficult TI was assessed by univariate analysis with the occurrence of severe and non-severe TIAEs and severe oxygen desaturation. A p value $<0.05$ was considered significant. Statistical analysis was performed using STATA V.14.0.

\section{RESULTS}

Data on 3023 TIs from 10 academic NICUs were collected during the study period. Of those, 1014 TIs did not meet inclusion criteria (figure 1). A total of 2009 TI encounters were analysed. Of those 2009 neonatal intubations, 276 (14\%) met the definition of difficult TI, and 1733 (86\%) did not meet the definition.

Patient characteristics are provided in table 1. Difficult intubations were more common in neonates $<32$ weeks corrected gestation and $<1500 \mathrm{~g}$ at the time of TI. The only comorbidity associated with difficult TI was sepsis $(p=0.02)$. Indications for intubation associated with difficult TIs included intubation for surfactant administration $(p=0.02)$ and intubation for airway obstruction $(\mathrm{p}=0.03)$.

Provider and practice characteristics are provided in table 2. The most common first providers to perform TI in neonates in the difficult TI group were neonatal fellows (48.9\%). The difficult TI group received less sedation/analgesia and paralytic medications prior to intubation as compared with non-difficult TI group $(p=0.03$ and $p<0.001)$. Videolaryngoscopy was used less commonly in the difficult TI group $(\mathrm{p}<0.001)$.

Neonates in the difficult TI group experienced more TIAEs and severe oxygen desaturations during intubation (table 3 ). In the difficult TI group, the OR for experiencing an adverse event was 4.9 (95\% CI 3.7 to $6.4 ; \mathrm{p}<0.0001)$ and the OR for severe desaturation was 4.1 (95\% CI 3.0 to $5.5 ; \mathrm{p}<0.001)$.

The value of bedside screening tests for difficult TI are provided in table 4. Upper airway obstruction, a known history of difficult airway, micrognathia, limited mouth opening, limited neck extension, cleft palate and short thyromental distance were

Table 1 Patient characteristics stratified by the difficulty of intubation

\begin{tabular}{|c|c|c|c|}
\hline Patient characteristics & $\begin{array}{l}\text { Non-difficult intubation } \\
(\mathrm{n}=1733)\end{array}$ & $\begin{array}{l}\text { Difficult intubation } \\
(\mathrm{n}=276)\end{array}$ & $P$ value \\
\hline Gestational age (GA) at birth, weeks, median $(25 \%, 75 \%)$ & $29(25,35)$ & $28(25,34)$ & 0.08 \\
\hline Birth weight, g, median $(25 \%, 75 \%)$ & $1090(710,2515)$ & $904(680,2020)$ & 0.005 \\
\hline Day of life at time of intubation, median $(25 \%, 75 \%)$ & $14(1,50)$ & $10(1,41)$ & 0.09 \\
\hline Corrected GA at time of intubation, weeks, median $(25 \%, 75 \%)$ & $34(29,39)$ & $31(28,37)$ & $<0.001$ \\
\hline Weight at time of intubation, $\mathrm{g}$ & $1870(1000,3050)$ & $1326(830,2600)$ & $<0.001$ \\
\hline Sex* (male), n (\%) & $1024(59)$ & $158(57)$ & 0.75 \\
\hline \multicolumn{4}{|l|}{ Comorbidities, $\mathrm{n}(\%)$} \\
\hline Acute respiratory failure & $1003(58)$ & $171(62)$ & 0.2 \\
\hline Chronic respiratory failure & $412(24)$ & $66(24)$ & 0.9 \\
\hline Congenital anomaly requiring surgery & $176(10)$ & $19(7)$ & 0.1 \\
\hline Neurological impairment (eg, HIE, seizures and stroke) & $125(7)$ & $21(8)$ & 0.4 \\
\hline Cardiac disease (excluding isolated patent ductus arteriosus) & $124(7)$ & $19(7)$ & 1.0 \\
\hline Airway or craniofacial anomaly & $110(6)$ & $20(7)$ & 0.6 \\
\hline Sepsis & $106(6)$ & $28(10)$ & 0.02 \\
\hline Surgery/procedure for acquired disorder & $7(<1)$ & $3(1)$ & 0.2 \\
\hline \multicolumn{4}{|l|}{ Indication for intubation, $\mathrm{n}(\%)$} \\
\hline Ventilation failure & $664(38)$ & $94(34)$ & 0.2 \\
\hline Oxygenation failure & $550(32)$ & $71(29)$ & 0.3 \\
\hline Frequent apnoea and desaturations & $343(20)$ & $54(20)$ & 1.0 \\
\hline Surfactant administration & $299(17)$ & $64(23)$ & 0.02 \\
\hline Intubation for procedure & $194(11)$ & $20(7)$ & 0.06 \\
\hline Upper airway obstruction & $87(5)$ & $23(8)$ & 0.03 \\
\hline Unstable haemodynamics & $37(2)$ & $3(1)$ & 0.3 \\
\hline Absent protective reflexes & $8(<1)$ & $4(2)$ & 0.07 \\
\hline Neuromuscular weakness & $4(<1)$ & $1(<1)$ & 0.5 \\
\hline
\end{tabular}

*Sex was unknown in four neonates.

HIE, hypoxic-ischaemic encephalopathy. 


\begin{tabular}{|c|c|c|c|}
\hline Provider and practice characteristic & $\begin{array}{l}\text { Non-difficult } \\
\text { intubation } \\
(n=1733)\end{array}$ & $\begin{array}{l}\text { Difficult } \\
\text { intubation } \\
(n=276)\end{array}$ & $P$ value \\
\hline \multicolumn{4}{|l|}{ First intubator } \\
\hline Neonatology fellow, $\mathrm{n}(\%)$ & $474(27)$ & $135(49)$ & $<0.001$ \\
\hline $\begin{array}{l}\text { Nurse practitioner/physician } \\
\text { assistant/hospitalist }\end{array}$ & $698(40)$ & $67(24)$ & \\
\hline Paediatric resident & $266(15)$ & $34(12)$ & \\
\hline Neonatology attending & $122(7)$ & $18(6)$ & \\
\hline Respiratory therapist & $44(3)$ & $0(0)$ & \\
\hline Other & $129(7)$ & $22(8)$ & \\
\hline \multicolumn{4}{|l|}{ Device used } \\
\hline Direct laryngoscope & $1353(78)$ & $230(83)$ & $<0.001$ \\
\hline Videolaryngoscope & $380(30)$ & $35(13)$ & \\
\hline Other & $0(0)$ & $11(4)$ & \\
\hline \multicolumn{4}{|l|}{ Medications given } \\
\hline Analgesic/sedative & $1125(65)$ & $161(58)$ & 0.03 \\
\hline Paralytic* & $865(50)$ & $81(23)$ & $<0.001$ \\
\hline
\end{tabular}

${ }^{*}$ Ten neonates were reported to receive paralysis only. From the data, it is unclear whether these neonates were already sedated before TI procedure was initiated. $\mathrm{Tl}$, tracheal intubation.

all more common in the difficult TI group $(\mathrm{p}=0.03$ to $<0.001)$. The sensitivity, positive predictive value and area under the ROC for all screening tests of difficult TI were poor.

\section{DISCUSSION}

We analysed the NEAR4NEOS database to determine the incidence of difficult TIs in the NICU, quantify the clinical impact of difficult TIs and examine the utility of bedside predictors of difficult neonatal TI. We found that $14 \%$ of intubations in the NICU met our definition of 'difficult'. Difficult TIs were associated with the higher occurrence of adverse events and oxygen desaturations. Bedside screening tests of difficult TI had little value to predict the occurrence of a difficult TI. To the authors' knowledge, this is the first report to examine difficult TI in the NICU.

The first aim of this study was to determine the incidence of difficult TIs in the NICU. We found that 14\% (276 of 2009) of intubations in the NICUs we studied were difficult TIs. This incidence is higher than in prior multicentre studies reports from the adult and paediatric ICU. ${ }^{16}{ }^{17}$ We believe this difference fundamentally comes from our study population. Inherently, the airway features in neonatal population are different from adult and paediatric population. However, we also found that difficult TIs were more frequently performed by fellows and less frequently performed with video laryngoscopy and premedication. The association between fellows as first laryngoscopist and difficult TI may be explained by experience. Fellows, particularly early on, may have little experience in performing TIs in neonates, certainly less than that of a consultant/attending physician. Video laryngoscopy was only used in $13 \%$ of difficult TIs and in 30\% of non-difficult TIs. Video laryngoscopy has been associated with higher first attempt intubation success in trainees when compared with direct laryngoscopy. ${ }^{10}$ It is possible that higher use of video laryngoscopy in adult and paediatric TI studies may be associated with higher TI success and thus with lower incidence if difficult TI in their population. Future studies are needed whether video laryngoscopy will decrease number of TI attempts and difficult TIs. Analgesia/sedation was given in $65 \%$ of non-difficult intubations and $58 \%$ of difficult intubations; and paralytics were given in $50 \%$ and $23 \%$ of cases. This means that more than a third of the time, premedication was not used. Premedication has been associated with increase TI success rates. $^{2}$ Therefore, it is possible that the prevalence of difficult intubation could be-at least partly-explained by insufficient use of premedication. Given the higher incidence of difficult TI in the NICU, we believe that specific attention should be paid to improving neonatal TI safety. This includes careful consideration of the first laryngoscopist, the device and premedication. ${ }^{14}$

The second aim of this study was to examine the clinical impact of difficult neonatal TI. We found that TIs that met the definition of 'difficult' had more TIAEs and severe desaturations. In the difficult TI group, the OR for experiencing an adverse event was 4.9, and the OR for severe desaturation was 4.1. This suggests that the definition we used was clinically meaningful. We believe establishing this definition of difficult neonatal TI sets the stage for future studies in this area and may help NICUs develop quality improvement approaches to address difficult neonatal TIs. The Paediatric Difficult Intubation registry recommends that the number of laryngoscopy attempts is limited to two or less and advocates for quickly transitioning to indirect intubation techniques when direct laryngoscopy fails after two attempts. ${ }^{19}$ One of the NEAR4NEOS study sites has established a difficult airway pathway in which two failed TI attempts by an experienced practitioner triggers the activation of an airway response team that includes an anaesthesiologist, an otolaryngologist and a respiratory therapist. ${ }^{20}$

The third aim of this study was to examine the utility of bedside screening tests of difficult neonatal TI. We found that difficult TIs occurred more commonly in younger and smaller patients. Prior reports from the paediatric ICU have found similar results. ${ }^{17}$ The reason why younger and smaller patients are more difficult to intubate cannot be determined from the results of this study. However, the smaller size of the airway, proportionately larger head, more cephalad position of the larynx, proportionately larger tongue and the large and floppy epiglottis are all potential factors. ${ }^{2122}$

We found that the sensitivity, positive predictive value and area under the ROC for all the bedside screening tests

Table 3 Adverse events in neonates with difficult intubation*

\begin{tabular}{llllll}
\hline Adverse events & $\begin{array}{l}\text { Non-difficult intubation } \\
(\mathbf{n}=1733)\end{array}$ & $\begin{array}{l}\text { Difficult intubation } \\
(\mathbf{n}=\mathbf{2 7 6})\end{array}$ & P value & Risk ratio (95\% Cl) & OR (95\% Cl) \\
\hline Any tracheal intubation adverse event, $\mathrm{n}(\%)$ & $241(14)$ & $122(44)$ & $<0.001$ & $3.2(2.7$ to 3.8$)$ & $4.9(3.7$ to 6.4$)$ \\
Severe tracheal intubation adverse events, $\mathrm{n}(\%)$ & $51(3)$ & $36(13)$ & $<0.001$ & $4.4(2.9$ to 6.6$)$ & $4.9(3.2$ to 7.7$)$ \\
Non-severe tracheal intubation adverse events, $\mathrm{n}(\%)$ & $210(12)$ & $104(38)$ & $<0.001$ & $3.1(2.6$ to 3.8$)$ & $4.4(3.3$ to 5.8$)$ \\
Severe desaturation, $\mathrm{n}(\%)+$ & $732(42)$ & $207(75)$ & $<0.001$ & $1.7(1.5$ to 1.9$)$ & $4.1(3.0$ to 5.5$)$ \\
\hline
\end{tabular}

* Severe desaturation was defined as a drop in $\mathrm{SpO}_{2}$ by $>20 \%$ from highest $\mathrm{SpO}_{2}$ before intubation to lowest $\mathrm{SpO}_{2}$ during intubation attempt.

tDifficult intubation was defined as three or more failed intubation attempts by a non-resident provider. 
Table 4 Relative value of bedside screening tests of difficult intubation

\begin{tabular}{|c|c|c|c|c|c|c|c|c|}
\hline $\begin{array}{l}\text { Screening tests of difficult } \\
\text { intubation }\end{array}$ & $\begin{array}{l}\text { Non-difficult } \\
\text { intubation } \\
(n=1733)\end{array}$ & $\begin{array}{l}\text { Difficult } \\
\text { intubation } \\
(n=276)\end{array}$ & $P$ value & $\begin{array}{l}\text { Sensitivity } \\
(\%, 95 \% \mathrm{Cl})\end{array}$ & $\begin{array}{l}\text { Specificity } \\
(\%, 95 \% \mathrm{CI})\end{array}$ & $\begin{array}{l}\text { Positive } \\
\text { predictive value } \\
(\%, 95 \% \mathrm{Cl})\end{array}$ & $\begin{array}{l}\text { Negative } \\
\text { predictive value } \\
(\%, 95 \% \mathrm{Cl})\end{array}$ & $\begin{array}{l}\text { ROC area } \\
(95 \% \mathrm{Cl})\end{array}$ \\
\hline Upper airway obstruction, $\mathrm{n}(\%)$ & $61(4)$ & $28(10)$ & $<0.001$ & $10(7$ to 14$)$ & 97 (96 to 97 ) & $32(22$ to 42$)$ & 87 (86 to 89 ) & $0.53(0.52$ to 0.55$)$ \\
\hline Known prior history of difficult airway & $150(9)$ & $41(15)$ & 0.001 & $15(11$ to 20$)$ & 91 (90.9 to 93) & 22 (16 to 28$)$ & 87 (85 to 88 ) & $0.53(0.51$ to 0.55$)$ \\
\hline Micrognathia & $27(2)$ & $12(4)$ & 0.002 & 4 (2 to 8$)$ & 98 (98 to 99$)$ & 31 (17 to 48$)$ & 87 (85 to 88 ) & $0.51(0.5$ to 0.53$)$ \\
\hline Limited mouth opening & $262(15)$ & $60(21)$ & 0.005 & 15 (14 to 17$)$ & 78 (73 to 83$)$ & $13(11$ to 15$)$ & 81 (77 to 86$)$ & $0.47(0.44$ to 0.49$)$ \\
\hline Limited neck extension & $27(2)$ & $10(4)$ & 0.02 & $4(2$ to 7$)$ & 98 (98 to 99$)$ & 27 (14 to 44$)$ & 87 (85 to 88 ) & $0.51(0.49,0.52)$ \\
\hline Cleft palate & $17(1)$ & $7(3)$ & 0.03 & $3(1$ to 5$)$ & $99(98,99)$. & 29 (13 to 51$)$ & 86 (85 to 88 ) & 0.51 (0.49 to 0.52$)$ \\
\hline Midfacial hypoplasia & $10(<1)$ & $4(1)$ & 0.1 & $2(0.4$ to 4$)$ & 99 (98 to 99$)$ & 29 (8 to 58$)$ & 86 (85 to 88 ) & $0.5(0.49$ to 0.51$)$ \\
\hline Short thyromental distance & $250(14)$ & $43(16)$ & 0.6 & 14 (13 to 16$)$ & 84 (80 to 88$)$ & $14(12$ to 15$)$ & 85 (81 to 89$)$ & $0.49(0.47$ to 0.52$)$ \\
\hline
\end{tabular}

ROC, receiver operator curve.

of difficult TI we studied were poor. Therefore, their clinical utility is questionable. The screening tests we chose were based on those used in paediatric and adult patients. This is the first report of using these screening tests in neonates. As they were not developed specifically for neonates, there is some inherent difficulty in applying the screening tests in the neonatal population, particularly in very preterm babies. However, the lack of consistent strong predictors for difficult TI is also an issue with paediatric and adult TI. ${ }^{22-24}$ A recent Cochrane review of adult TI cautioned that 'standard bedside airway examination tests should be interpreted with caution, as they do not appear to be good screening tests'. ${ }^{24}$ Based on our results, we suggest that gestational age, weight, indication for intubation and bedside screening tests of difficult TI all be considered when deciding who will perform an intubation in the NICU. This is especially important in extremely premature neonates where failed intubations have been associated with intraventricular haemorrhage and impaired neurodevelopmental outcomes. ${ }^{12}{ }^{13}$ Based on the risk of difficult TI in small premature neonates, and the potential for adverse events, it seems prudent to have more experienced providers intubate those patients. Consideration should also be given to using videolaryngoscopy and premedication with paralysis as these have both been linked to greater first attempt success and fewer adverse events. ${ }^{10112526}$

This study has several limitations. First, some readers may not support our definition of difficult TI as one requiring three or more intubation attempts by a non-resident provider. Some would argue that a neonatal fellow early in training, or a new nurse practitioner, is still a novice intubator and no different than a resident. We believe that creating any arbitrary cut point of experience (eg, fellows with 6 months of training) has inherent limitations. The definition we used is the same as that used in the adult and paediatric ICU. ${ }^{16}{ }^{17}$ We chose to use that definition to ensure a consistent definition across studies and the ability to allow analysis across the different ICU populations. Second, the intubation data used in this analysis was self-reported by the medical teams at the time of intubation. This places our results at risk for reporting bias. Third, the bedside screening tests for difficult TI were determined by the medical teams at the time of the intubation. Therefore, the accuracy of the assessments of each screening test was dependent on the team's review of the patient's history and accurate assessment of the physical exam features. Inaccurate determination of the screening tests could bias our results. Third, this study was conducted on a cohort of neonates from 10 academic (non-community) NICUs, and neonates intubated in the delivery room were not included. This may limit the generalisability of our findings. Fourth, the data here represents the number of TIs that met our definition, not the number of neonates. It is possible that some neonates were repeatedly intubated and were counted more than once in this study dataset. Finally, we were not able to obtain long-term data on the outcomes of neonates with and without difficult TIs. Additional studies are needed to determine the impact of difficult TI on long-term neonatal outcomes.

\section{CONCLUSION}

The occurrence of difficult TI in the NICU was 14\%, which is higher than in the paediatric and adult ICUs. The occurrence of difficult TIs was strongly associated with adverse events and severe oxygen desaturations. Difficult TIs occurred more common in small premature neonates. The ability of bedside screening tests to predict difficult TI was poor. These results suggest that when intubating neonates, healthcare teams should carefully consider who will perform TI, as well as what medications and devices should be used in order to improve neonatal TI safety.

\section{Author affiliations}

${ }^{1}$ Department of Pediatrics, University of Washington, Seattle, Washington, USA

${ }^{2}$ Department of Pediatrics, Division of Neonatology, Children's Hospital of

Philadelphia, Philadelphia, Pennsylvania, USA

${ }^{3}$ Department of Pediatrics, University of Pennsylvania School of Medicine,

Philadelphia, Pennsylvania, USA

${ }^{4}$ Department of Pediatrics, Centre Hospitalier Universitaire Sainte-Justine, Montréal, Sainte-Justine, Canada

${ }^{5}$ Nursing and Respiratory Care, Children's Hospital of Philadelphia, Philadelphia, Pennsylvania, USA

${ }^{6}$ Department of Pediatrics, Penn State - Hershey, Hershey, Pennsylvania, USA

${ }^{7}$ Yale New Haven Health System, New Haven, Connecticut, USA

${ }^{8}$ University Hospital Schleswig-Holstein, Department of Pediatrics, Luebeck, Germany ${ }^{9}$ Department of Pediatrics, Dartmouth-Hitchcock Health System, Lebanon, New Hampshire, USA

${ }^{10}$ Department of Neonatology, KK Women's and Children's Hospital, Singapore ${ }^{11}$ Department of Pediatrics, University of Colorado Denver School of Medicine, Aurora, Colorado, USA

${ }^{12}$ Department of Pediatrics, WakeMed Health and Hospitals, Raleigh, North Carolina, USA

${ }^{13}$ Department of Pediatrics, University of Washington, Seattle, USA

${ }^{14}$ Department of Pediatrics, University of California San Diego Medical Center, San Diego, California, USA

${ }^{15}$ Anesthesiology and Critical Care Medicine, Children's Hospital of Philadelphia,

Philadelphia, Pennsylvania, USA

${ }^{16}$ Center for Simulation, Advanced Education, and Innovation, Children's Hospital of Philadelphia, Philadelphia, Pennsylvania, USA

Acknowledgements The authors would like to thank the healthcare teams at participating National Emergency Airway Registry for Neonates (NEAR4NEOS) sites for their diligent completion of NEAR4NEOS data collection forms. We would also like to thank Hayley Buffman at Children's Hospital of Philadelphia for her work with the NEAR4NEOs database.

Collaborators The NEAR4NEOS. 
Contributors The manuscript has been seen and approved by all authors and they have taken due care to ensure the integrity of the work. All authors have made substantial contributions to all of the following: (1) the conception and design of the manuscript, (2) drafting the article or revising it critically for important intellectual content and (3) final approval of the version to be submitted.

Funding TS is supported by NICHD 1R21HD091687 and 1R21HD089151. AN is supported by NICHD 1R21HD089151, AHRQ R18HS02264, R18HS024511. EEF is supported by an NICHD Career Development Award K23HD084727.

Competing interests None declared.

Patient consent for publication Not required.

Provenance and peer review Not commissioned; externally peer reviewed.

\section{REFERENCES}

1 Sawyer T, French H, Ades A, et al. Neonatal-perinatal medicine fellow procedural experience and competency determination: results of a national survey. J Perinatol 2016:36:570-4.

2 Foglia EE, Ades A, Napolitano N, et al. Factors associated with adverse events during tracheal intubation in the NICU. Neonatology 2015;108:23-9.

3 Hatch LD, Grubb PH, Lea AS, et al. Endotracheal intubation in neonates: a prospective study of adverse safety events in 162 infants. J Pediatr 2016;168:62-6.

4 Leone TA, Rich W, Finer NN. Neonatal intubation: success of pediatric trainees. J Pediatr 2005;146:638-41.

5 Falck AJ, Escobedo MB, Baillargeon JG, et al. Proficiency of pediatric residents in performing neonatal endotracheal intubation. Pediatrics 2003;112:1242-7.

6 Bismilla Z, Finan E, McNamara PJ, et al. Failure of pediatric and neonatal trainees to meet Canadian Neonatal Resuscitation Program standards for neonatal intubation. J Perinatol 2010;30:182-7.

7 O'Donnell CP, Kamlin CO, Davis PG, et al. Endotracheal intubation attempts during neonatal resuscitation: success rates, duration, and adverse effects. Pediatrics 2006;117:e16-21.

8 Downes KJ, Narendran V, Meinzen-Derr J, et al. The lost art of intubation: assessing opportunities for residents to perform neonatal intubation. J Perinatol 2012;32:927-32.

9 Haubner LY, Barry JS, Johnston LC, et al. Neonatal intubation performance: room for improvement in tertiary neonatal intensive care units. Resuscitation 2013:84:1359-64.

10 O'Shea JE, Thio M, Kamlin CO, et al. Videolaryngoscopy to teach neonatal intubation: a randomized trial. Pediatrics 2015;136:912-9.
11 Pouppirt NR, Nassar R, Napolitano N, et al. Association between video laryngoscopy and adverse tracheal intubation-associated events in the neonatal intensive care unit. J Pediatr 2018;201:281-4.

12 Sauer $\mathrm{CW}$, Kong JY, Vaucher YE, et al. Intubation attempts increase the risk for severe intraventricular hemorrhage in preterm infants-a retrospective cohort study. $J$ Pediatr 2016:177:108-13

13 Wallenstein MB, Birnie KL, Arain YH, et al. Failed endotracheal intubation and adverse outcomes among extremely low birth weight infants. J Perinatol 2016;36:112-5.

14 Sawyer T, Foglia E, Hatch LD, et al. Improving neonatal intubation safety: a journey of a thousand miles. J Neonatal Perinatal Med 2017;10:125-31.

15 Lynch TA, Glantz JC, Drennan K. Prenatal prediction of difficult intubation in periviable neonates using standard fetal biometric parameters. Am J Perinatol 2018.

16 De Jong A, Molinari N, Terzi N, et al. Early identification of patients at risk for difficult intubation in the intensive care unit: development and validation of the MACOCHA score in a multicenter cohort study. Am J Respir Crit Care Med 2013;187:832-9.

17 Graciano AL, Tamburro R, Thompson AE, et al. Incidence and associated factors of difficult tracheal intubations in pediatric ICUs: a report from national emergency airway registry for children: NEAR4KIDS. Intensive Care Med 2014;40:1659-69.

18 Harris PA, Taylor R, Thielke R, et al. Research electronic data capture (REDCap)-a metadata-driven methodology and workflow process for providing translational research informatics support. J Biomed Inform 2009;42:377-81.

19 Fiadjoe JE, Nishisaki A, Jagannathan $\mathrm{N}$, et al. Airway management complications in children with difficult tracheal intubation from the Pediatric Difficult Intubation (PeDI) registry: a prospective cohort analysis. Lancet Respir Med 2016;4:37-48.

20 Nishisaki A, Giordano R, Lavelle J, et al. 20Children's hospital of Philadelphia, difficult/

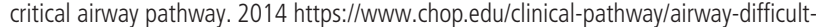
critical-clinical-pathway

21 Belanger J, Kossick M. Methods of identifying and managing the difficult airway in the pediatric population. Aana J 2015;83:35-41

22 aggarwal $A$, chand verma uttam. Evaluation of difficult airway predictors in pediatric population as a clinical investigation. J Anesth Clin Res 2012;03:1-5.

23 Vannucci A, Cavallone LF. Bedside predictors of difficult intubation: a systematic review. Minerva Anestesiol 2016;82:69-83.

24 Roth D, Pace NL, Lee A, et al. Airway physical examination tests for detection of difficult airway management in apparently normal adult patients. Cochrane Database Syst Rev 2018;5:CD008874

25 Krick J, Gray M, Umoren R, et al. Premedication with paralysis improves intubation success and decreases adverse events in very low birth weight infants: a prospective cohort study. J Perinatol 2018;38:681-6.

26 Colm Patrick Finbarr ODonnell. Intubation difficulty in neonatology: are you experienced? Arch Dis Child 2019;104:458-60. 